\title{
Large-scale Integration of Multidimensional Quantum Photonics Circuits on Silicon
}

\author{
Wang, J.; Paesani, S.; Ding, Y.; Santagati, R.; Skrzypczyk, P.; Salavrakos, A.; Tura, J.; Augusiak, R.; \\ Mancinska, L.; Bacco, Davide
}

Total number of authors:

19

\section{Published in:}

Proceedings of 2018 Conference on Lasers and Electro-Optics

Link to article, DOI:

10.1364/CLEO_AT.2018.JTh5B.4

Publication date:

2018

Document Version

Peer reviewed version

Link back to DTU Orbit

Citation (APA):

Wang, J., Paesani, S., Ding, Y., Santagati, R., Skrzypczyk, P., Salavrakos, A., Tura, J., Augusiak, R., Mancinska, L., Bacco, D., Bonneau, D., Silverstone, J., Gong, Q., Acin, A., Rottwitt, K., Oxenløwe, L. K., O'Brien, J., Laing, A., \& Thompson, M. K. (2018). Large-scale Integration of Multidimensional Quantum Photonics Circuits on Silicon. In Proceedings of 2018 Conference on Lasers and Electro-Optics (pp. 1-2). Optical Society of America. https://doi.org/10.1364/CLEO_AT.2018.JTh5B.4

\section{General rights}

Copyright and moral rights for the publications made accessible in the public portal are retained by the authors and/or other copyright owners and it is a condition of accessing publications that users recognise and abide by the legal requirements associated with these rights.

- Users may download and print one copy of any publication from the public portal for the purpose of private study or research.

- You may not further distribute the material or use it for any profit-making activity or commercial gain

- You may freely distribute the URL identifying the publication in the public portal 


\title{
Large-scale Integration of Multidimensional Quantum Photonics Circuits on Silicon
}

\author{
J. Wang ${ }^{1,2}$, S. Paesani ${ }^{1}$, Y. Ding ${ }^{3}$, R. Santagati ${ }^{1}$, P. Skrzypczyk $^{4}$, A. Salavrakos ${ }^{5}$,

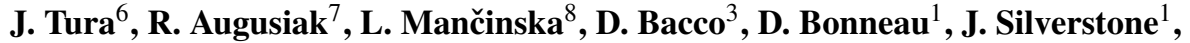 \\ Q. Gong ${ }^{2}$, A. Acín ${ }^{5,9}$, K. Rottwitt ${ }^{3}$, L. Oxenløwe ${ }^{3}$, J. O’Brien ${ }^{1}$, A. Laing ${ }^{1}$, M. Thompson ${ }^{1}$ \\ ${ }^{1}$ Quantum Engineering Technology Labs, H. H. Wills Physics Laboratory and Department of Electrical and \\ Electronic Engineering, University of Bristol, BS8 1FD, Bristol, United Kingdom \\ ${ }^{2}$ State Key Laboratory for Mesoscopic Physics, School of Physics, Collaborative Innovation Center of Quantum \\ Matter, Peking University, Beijing 100871, China \\ ${ }^{3}$ Centre for Silicon Photonics for Optical Communications (SPOC), DTU Fotonik, Technical University of Denmark, \\ Kgs. Lyngby, DK-2800, Denmark \\ ${ }^{4}$ H. H. Wills Physics Laboratory, University of Bristol, BS8 1TL, Bristol, United Kingdom \\ 5 ICFO-Institut de Ciencies Fotoniques, The Barcelona Institute of Science and Technology, 08860 Castelldefels, \\ Barcelona, Spain \\ ${ }^{6}$ Max-Planck-Institut für Quantenoptik, Hans-Kopfermann-Straße 1, 85748 Garching, Germany \\ ${ }^{7}$ Center for Theoretical Physics, Polish Academy of Sciences, Aleja Lotników 32/46, 02-668 Warsaw, Poland \\ ${ }^{8}$ QMATH, Department of Mathematical Sciences, University of Copenhagen, Universitetsparken 5, 2100 \\ Copenhagen $\emptyset$, Denmark \\ ${ }^{9}$ ICREA - Institució Catalana de Recerca i Estudis Avançats, Pg. Lluís Companys 23 Barcelona, 08010, Spain \\ Please correspondence to: yudin@fotonik.dtu.dk and mark.thompson@bristol.ac.uk
}

\begin{abstract}
We report the first large-scale silicon-photonics quantum circuit, integrating more than 500 photonic components, able to generate, manipulate and measure multidimensional entanglement fully on-chip with unprecedented precision, controllability and universality. C 2018 The Author(s) OCIS codes: $270.5585,130.0130$
\end{abstract}

\section{Introduction}

Multidimensional quantum systems exhibit distinct quantum properties and offer improvements in key applications, such as increasing capacity in quantum communication [1], strengthening quantum correlations [2], and enriching quantum simulation and computing schemes [3]. Photons represent a promising platform able to naturally encode and process qudits in various degrees of freedom, e.g., orbital angular momentum, temporal bin and frequency [4,5]. However, these approaches present limitations in terms of controllability, precision, universality and a full integration of elements, which represent bottlenecks for further developments of multidimensional quantum photonic technologies.

We report a large-scale integrated quantum photonics circuit in the silicon-on-insulator platform able to create, control and analyze on-chip multidimensional entanglement up to dimensions $15 \times 15$. Path-encoded qudits are obtained, where each photon exist over $d$ spatial modes simultaneously, and entanglement is produced by an excitation of an array of $d$ identical integrated photon-pair sources. Our chip enables the generation of multidimensional entangled states with an arbitrary degree of entanglement, and arbitrary multidimensional measurements with very high fidelity, verified by quantum state tomographies, Bell violations and other quantum protocols.

\section{Results}

Silicon quantum photonics offers intrinsic stability, high precision and dense integration [6]. We manufactured a largescale silicon quantum photonic circuit for multidimensional entanglement (Figure 1A and inset). A multidimensional entangled state $|\Psi\rangle=\sum_{k=0}^{d-1} c_{k}|k\rangle|k\rangle$ is produced by a coherent excitation of an array of $d$ identical photon-pair sources, where $c_{k}$ can be arbitrarily chosen by controlling the pump distribution through a network of MZIs before the sources, and $|k\rangle(k=0, \ldots, d-1)$ represents the logical state having a photon in its $k$-th optical mode. A network of asymmetric MZIs and crossers is used to separate the photons, and a triangular network of MZIs and phase-shifters is used to perform arbitrary local projective measurements on the qudits. The high indistinguishability of photon sources and high fidelity of qudit projectors enable the high-quality generation, control and analysis of multidimensional entanglement. We used quantum compressed sensing techniques to reconstruct bipartite entangled states. Figure 1B shows the reconstructed density matrix for a 12-dimensional entangled state having a high fidelity of $81 \%$, showing a significant improvement of the quality for multidimensional entanglement.

Multidimensional entanglement can be verified by the violation of generalized Bell inequalities $\tilde{I}_{d} \leq C_{d}$, where $\tilde{I}_{d}$ is a function of the joint probabilities, and $C_{d}$ is the classical bound for LHV models. We study a Bell-type inequalities, 

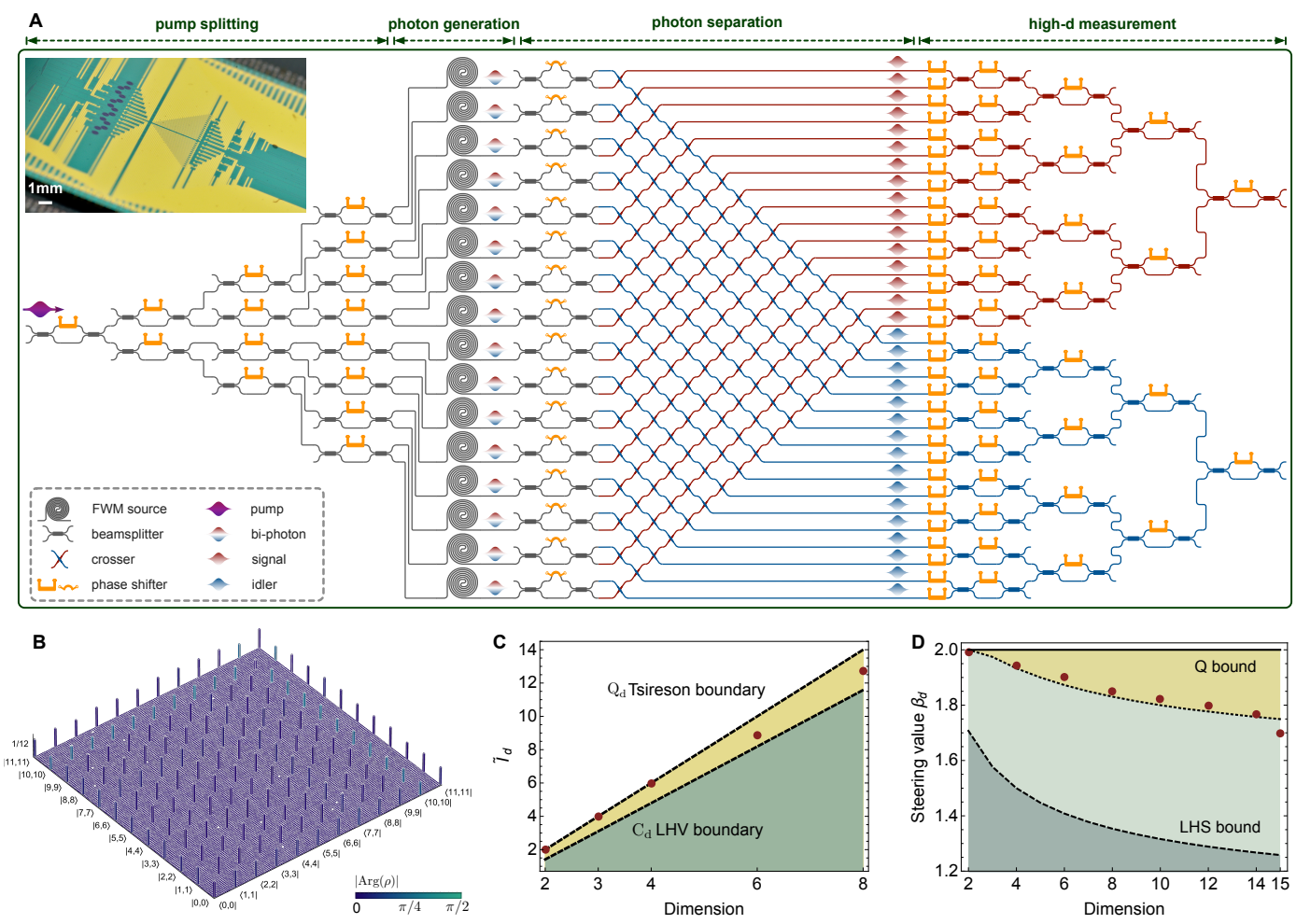

Fig. 1. A. Diagram for the integrated multidimensional quantum device. It integrates 16 spontaneous four-wave mixing photon-pair sources, 93 phase-shifters, 122 beamsplitters, 256 crossers and 64 grating couplers. A photon pair is generated in superposition across 16 optical modes, producing a multidimensional bipartite entangled state. The photons are separated by a network of asymmetric MZIs and crossers. Using a network of MZIs, we perform arbitrary local projective measurements on the entangled qudits. B. Reconstructed density matrix for a $d$-dimensional entangled state with a local dimension of 12. C. Bell violations, and D. EPR steering violations on $d$-dimensional entangled states. Red points are measured Bell values $\tilde{I}_{d}$ and steering values $\beta_{d}$, respectively.

that is tailored to obtain a maximal violation for maximally entangled qudit states [7]. The maximum value of $\tilde{I}_{d}$ obtainable with quantum states is given by $Q_{d}$, namely the Tsirelson bound. Figure 1C shows the measured Bell values for dimensions 2 to 8 . In all cases the classical bound is violated. In particular in dimensions 2 to 4 a strong violation is observed, closely approaching the $Q_{d}$ bound. Moreover, we obtained strong violations of the EPR steering inequalities for the 2 to 15 -dimensional states, verifying the presence of $d$-dimensional entanglement up to 15 in the one-sided device-independent scenario, see Fig. 1D. The high precision and controllability of our technology enable the experimental demonstration of unexplored applications, such as quantum randomness expansion and self-testing on multidimensional states.

In summary, we have demonstrated the first large-scale silicon-photonic quantum circuit comprising more than 500 elements, and enabling high-quality multidimensional entanglement up to dimensions $15 \times 15$.

\section{References}

1. Y. Ding et al., npj Quantum Information 3, 25 (2017).

2. H. M. Wiseman, S. J. Jones, A. C. Doherty, Phys. Rev. Lett. 98, 140402 (2007).

3. B. P. Lanyon et al., Nat. Phys. 5, 134140 (2009).

4. A. Mair, A. Vaziri, G. Weihs, A. Zeilinger, Nature 412, 313316 (2001).

5. M. Kues et al., Nature 546, 622626 (2017).

6. J. Wang et al., Nat. Phys., 13, 551-555 (2017).

7. A. Salavrakos et al., Phys. Rev. Lett. 119, 040402 (2017). 